\title{
Formation and stability of oil-in-water nanoemulsions containing rice bran oil: in vitro and in vivo assessments
}

\author{
Daniela S Bernardi ${ }^{1 *}$, Tatiana A Pereira ${ }^{1}$, Naira R Maciel ${ }^{1}$, Josiane Bortoloto ${ }^{1,2}$, Gisely S Viera ${ }^{1}$, Gustavo C Oliveira ${ }^{1}$ \\ and Pedro A Rocha-Filho ${ }^{1}$
}

\begin{abstract}
Background: Nanoemulsions have practical application in a multitude of commercial areas, such as the chemical, pharmaceutical and cosmetic industries. Cosmetic industries use rice bran oil in sunscreen formulations, anti ageing products and in treatments for skin diseases. The aim of this study was to create rice bran oil nanoemulsions using low energy emulsification methods and to evaluate their physical stability, irritation potential and moisturising activity on volunteers with normal and diseased skin types.
\end{abstract}

Results: The nanoemulsion developed by this phase diagram method was composed of $10 \%$ rice bran oil, $10 \%$ surfactants sorbitan oleate/PEG-30 castor oil, $0.05 \%$ antioxidant and $0.50 \%$ preservatives formulated in distilled water. The nanoemulsion was stable over the time course of this study. In vitro assays showed that this formulation has a low irritation potential, and when applied to human skin during in vivo studies, the nanoemulsion improved the skin's moisture and maintained normal skin pH values.

Conclusion: The results of irritation potential studies and in vivo assessments indicate that this nanoemulsion has potential to be a useful tool to treat skin diseases, such as atopic dermatitis and psoriasis.

\section{Background}

Nanoemulsions are obtained when the size of an emulsion globule reaches approximately $20-500 \mathrm{~nm}$. The small droplet size can resist the physical destabilisation caused by gravitational separation, flocculation and/or coalescence. It also avoids the creaming process because the droplet's Brownian motion is enough to overcome the gravitational separation force $[1,2]$. The size and polydispersity of nanoemulsions can affect properties such as particle stability, rheology, appearance, colour, texture and shelf life [3]. In nanoemulsions, the most frequent instability phenomenon is Ostwald ripening [4,5], which can be calculated according to the Lifshitz-Slezov and Wagner theory (LSW) using the following equation $[6,7]$ :

\footnotetext{
* Correspondence: danibernardi81@yahoo.com.br

${ }^{1}$ Departamento de Ciências Farmacêuticas, Faculdade de Ciências Farmacêuticas de Ribeirão Preto, Universidade de São Paulo, Ribeirão Preto, São Paulo, Brazil

Full list of author information is available at the end of the article
}

$$
\omega=\frac{d r^{3} n}{d t}=k \frac{D c_{\infty} \gamma M}{\rho^{2} R T}
$$

Where $\omega$ is defined as the rate of change of the cube of the number average radius, $D$ is the diffusion coefficient of the dispersed oil phase in the aqueous phase, $\gamma$ is the interfacial tension between the two phases, $c_{\infty}$ is the bulk solubility of the oil in the water and $\rho$ is the oil density. $k$ is a constant that has the value of $8 / 9$ in the LSW.

Nanoemulsions are well characterised and are a promising drug delivery system with practical applications for pharmaceutical, cosmetic and chemical industry applications. They have been used in intravenous, oral and ocular drug administrations and have reduced drug side effects and improved the pharmacological effects of the drugs given $[8-10,4]$. Nanoemulsions are primarily produced either by high-energy emulsification (e.g., high-pressure homogenisation) or by low-energy emulsification (using physicochemical properties of the components) [11]. This work focuses on the latter method for nanoemulsion synthesis.

\section{Biomed Central}


Rice (Oryza sativa) bran oil has unsaponifiable fractions that contain high levels of antioxidant-rich components, such as tocopherols/tocotrienols and gamma-oryzanol, which could be useful for topical formulations [12]. The cosmetics industry has used rice bran oil in sunscreen formulations [13], in topical aging prevention products [14] and in treatments for skin diseases [15].

When skin is affected by diseases such as atopic dermatitis and psoriasis, it exhibits a compromised skin barrier function that causes increased transepidermal water loss [16-18]. Moisturisers can help improve the skin's function by relieving the cutaneous manifestations of these diseases [18-20]. Measuring the stratum corneum (SC) hydration degree gives important information about the biophysical properties and the function of the skin [21].

In vitro studies, such as the HET-CAM (Hen's Egg Test on the Chorioallantoic Membrane), are used to evaluate cosmetics products by immediately showing whether or not a solid or liquid substance irritates the hen's egg chorioallantoic membrane [22].

The aim of this study was to obtain nanoemulsions of rice bran oil and to evaluate their physical stability, irritating potential and in vivo moisturising activity.

\section{Materials and methods Materials}

Sorbitan oleate (HLB 4.3) and rice bran oil were kindly provided from Lipo do Brasil (Brazil). PEG-30 castor oil (HLB 11.7) was donated by Oxiteno (Brazil). The preservative DMDM Hydantoin (and) Iodopropynyl Butylcarbamate (Glydant ${ }^{\circledR}$ plus) was obtained from Chemyunion (Brazil), and the antioxidant Butyl Hydroxy Toluene (BHT) was purchased from Synth (Brazil).

\section{Preparation of nanoemulsion}

The following surfactant mixtures were evaluated to find a stable nanoemulsion formulation: polysorbate $80 /$ sorbitan oleate, polysorbate 60 /sorbitan oleate, ceteareth-20 OE/ ceteth-2, PEG-15 castor oil/sorbitan oleate, PEG-30 castor oil/sorbitan oleate and PEG-40 castor oil/sorbitan oleate. The PEG-30 castor oil/sorbitan oleate $\left(\mathrm{HLB}_{\text {resulting }}=8.0\right)$ was the only surfactant combination that resulted in a stable formulation and therefore was chosen for further study.

The phase diagram method was used with different concentration of constituents to result in 42 different emulsion formulations. All emulsions were prepared according the Emulsion Phase Inversion (EPI) method, where the water and oil phases were heated separately at $75^{\circ} \mathrm{C}$, the water phase was added into the oil phase (rice bran oil and surfactants) while stirring at $600 \mathrm{rpm}$ (Fisatom, 713-Dmodel, Brazil), and the mixture was then cooled to $25^{\circ} \mathrm{C}$ while stirring.

\section{Determination of nanoemulsion droplet size}

The mean droplet size and polydispersity index of the nanoemulsions were determined by dynamic light scattering (DLS) (Zetasizer, modelo ZS, Malvern Instruments, UK). Measurements were performed at $25^{\circ} \mathrm{C}$ using a scattering angle of $90^{\circ}$. Samples were considered polydisperse when the polydispersity index was higher than 0.2 [23].

\section{Stability study}

The preliminary stability of the nanoemulsion preparation was evaluated at 24 hours by centrifuge and thermal stress analyses. Stability was assessed by macroscopic emulsion observation and droplet size analysis. The purpose of these tests was to select a stable, low-surfactant formulation with a nanoemulsion-size droplet and stable physicochemical properties. The selected nanoemulsion was prepared in triplicate, and the samples were stored at $25 \pm 2^{\circ} \mathrm{C}, 40 \pm 2^{\circ} \mathrm{C}$ and $5 \pm 2^{\circ} \mathrm{C}$. Tests were performed at 24 hours, 7, 15, 30, 60 and 90 days after preparation. The analysis measurements were droplet size, $\mathrm{pH}$ value and electrical conductivity.

\section{Physical-chemical analyses}

To perform the thermal stress test, nanoemulsions were heated in an ultra bath thermostat (Nova Técnica, Brazil) at temperatures ranging from 40 to $80^{\circ} \mathrm{C}$. The temperature was increased by $5^{\circ} \mathrm{C}$ every 30 minutes. The nanoemulsions were centrifuged (Fanem 206-R, Brazil) at 1000, 2500 and $3500 \mathrm{rpm}$ (70, 440 and $863 \mathrm{G}$, respectively) for 15 minutes in each rotation to accelerate possible instability phenomena. A pH meter (PM608 model - Analion, Brazil) was used to measure the $\mathrm{pH}$ of the nanoemulsions at $25 \pm 2^{\circ} \mathrm{C}$. The electrical conductivity was evaluated at $25 \pm 2^{\circ} \mathrm{C}$ by a portable conductivity meter (mCA-150 model, Tecnopon, Brazil).

\section{Irritant test in an organotypic model - HET-CAM (Hen's Egg Test on the Chorioallantoic Membrane)}

The HET-CAM test is routinely used to evaluate the potential eye irritation of raw materials but can in some cases be used to evaluate skin irritation, e.g. in the case of surfactants. Irritation causes alterations in the vascular system of the HET-CAM that result in membrane discoloration, haemorrhaging and increased perfusion. The method used in this manuscript is a modification of the method described by Luepke [24] and adapted by Mehling [25] that allows the immediate evaluation of irritation by solid or liquid substances in the hen's egg chorioallantoic membrane. Each substance was tested on three fertilised eggs that were incubated for 9 days prior to testing. The CAM (Chorioallantoic Membrane) was exposed to $300 \mu \mathrm{L}$ of one of the following 
substances: (1) nanoemulsion ( $\mathrm{pH}$ 6.54), (2) surfactant solution blend (5\% sorbitan oleate, 5\% PEG-30 castor oil and $90 \%$ water ( $\mathrm{pH} 6.32$ ), (3) Sodium lauryl sulphate (SLS) 10\% w/w (positive control, $\mathrm{pH}$ 6.05) and (4) saline solution (negative control, $\mathrm{pH}$ 6.0). The CAM was rinsed with physiological saline solution after 30 seconds of exposure to each substance, and the intensity of the reactions (hyperaemia, haemorrhage and coagulation) was semi-quantitatively assessed on a scale of $0.5,2$ and 5 minutes after treatment; longer observation times give no additional important information. The numerical time-dependent scores for hyperaemia, haemorrhage and coagulation are summed to give a single numerical value indicating the irritation potential of the test substance on a scale with a maximum value of 21 . The mean value of four tests makes possible an assessment by a classification scheme analogous to the Draize categories (Table 1).

\section{In vivo assessment}

Approval to conducted the in vivo studies was obtained from the Ethics Committee of Faculdade de Ciências Farmacêuticas de Ribeirão Preto, Ribeirão Preto, São Paulo, Brazil, under protocol number CEP/FCFRP n 147.

Seventeen Caucasian volunteers with healthy skin (all women, 20-29 years old), five patients with atopic dermatitis (all women, 21-30 years old), and four psoriasis patients (1 man and 3 women, 28-56 years old) without skin lesions on the forearm were included in the study. The forearm area was washed with mild soap two hours before the analysis. The volunteers were kept for $10 \mathrm{~min}$ in a controlled room at ambient temperature $\left(25 \pm 2^{\circ} \mathrm{C} ; 29-34 \%\right.$ relative humidity) before starting the assay. Baseline values were determined using a Corneometer CM 820, a Sebumeter SM 810 and a Skin-pH-meter PH 900 instruments (Courage \&Khazaka, Köln, Germany) before applying the formulation. Then, $50 \mu \mathrm{L}$ of nanoemulsion was applied to the right forearm in three rectangular areas of $13.80 \mathrm{~cm}^{2}$ and distributed for 20 seconds by rubbing the test area using a circular motion. Excess nanoemulsion was left on the skin. The treated skin of each patient was measured 30, 60, 90, 120 and 150 minutes after nanoemulsion application, with one measure for sebum content and triplicate measurements of electrical capacitance and skin $\mathrm{pH}$.

Table 1 Classification of cumulative scores in the chorioallantoic membrane test (According Luepke 1985 [24])

\begin{tabular}{cc}
\hline Cumulative Score & Irritation assessment \\
\hline $0-0.9$ & Practically none \\
$1-4.9$ & Slight \\
$5-8.9$ & Moderate \\
$9-21$ & Strong \\
\hline
\end{tabular}

\section{Results and discussion}

\section{Preparation of nanoemulsion}

The phase diagram with the rice bran oil, surfactants sorbitan oleate/PEG-30 castor oil and water shows formation of five different areas: (I) O/W (Oil/Water) nanoemulsion, (II) phase separation, (III) gel phase, (IV) W/O (Water/ Oil) emulsions and (V) O/W emulsions (Figure 1).

Table 2 shows the composition and the droplet size of the nanoemulsion systems.

All of the nanoemulsions (Table 2) were stable when tested using the centrifugation test. The only formulation that showed signs of instability at high temperatures $\left(70^{\circ} \mathrm{C}\right.$ ) was the nanoemulsion composed of 10:20:70 (oil, surfactants and water, respectively).

A surfactant concentration of $5.00 \%$ was not sufficient to form a nanoemulsion, even with smaller amounts of oil. The surfactant amount affects the stabilisation and size of the emulsion droplets. From the experimental results, the nanoemulsion that used the lowest possible surfactant concentration while still maintaining thermal stability, centrifugal stability and small droplet size was selected as the working formulation.

Based on these preliminary results, we chose a formulation composed of $10 \%$ rice bran oil, $10 \%$ surfactant blend and $80 \%$ water and proceeded with further stability tests and in vitro and in vivo evaluations.

\section{Stability study}

The formulation composed of 10:10:80 (rice bran oil, surfactant blend and water) was supplemented with $0.05 \%$ antioxidants and $0.50 \%$ preservatives. The formulation was tested at three different storage temperatures: $25 \pm 2^{\circ} \mathrm{C}, 40 \pm 2^{\circ} \mathrm{C}$ and $5 \pm 2^{\circ} \mathrm{C}$. By granulometric analysis the particles maintained a mono-disperse, monomodal peak after 24 hours (Figure 2). The formulation was stable for up to 90 days as determined by macroscopic analysis.

Droplet size measurements are a good indicator of the formulation stability. A fast droplet size increase indicates low system stability. The droplet size for this formulation remained constant over 90 days for all temperature conditions (Figure 3).

The nanoemulsions had polydispersity index values below 0.2 throughout the 90-day testing period, indicating the high fidelity of the system (low polydispersity), which may reflect the overall stability of this formulation and synthesis method. Polydispersity values near 1.0 are indicative of a polydisperse system [26]. The long term stability of nanoemulsions was previously evaluated and was also verified by stability studies conducted over three months. The W/O nanoemulsion produced by low energy emulsification showed no difference in droplet size over the study period at both $25^{\circ} \mathrm{C}$ and $4^{\circ} \mathrm{C}$ [27]. The $\mathrm{W} / \mathrm{O}$ nanoemulsion demonstrated high physical stability, 


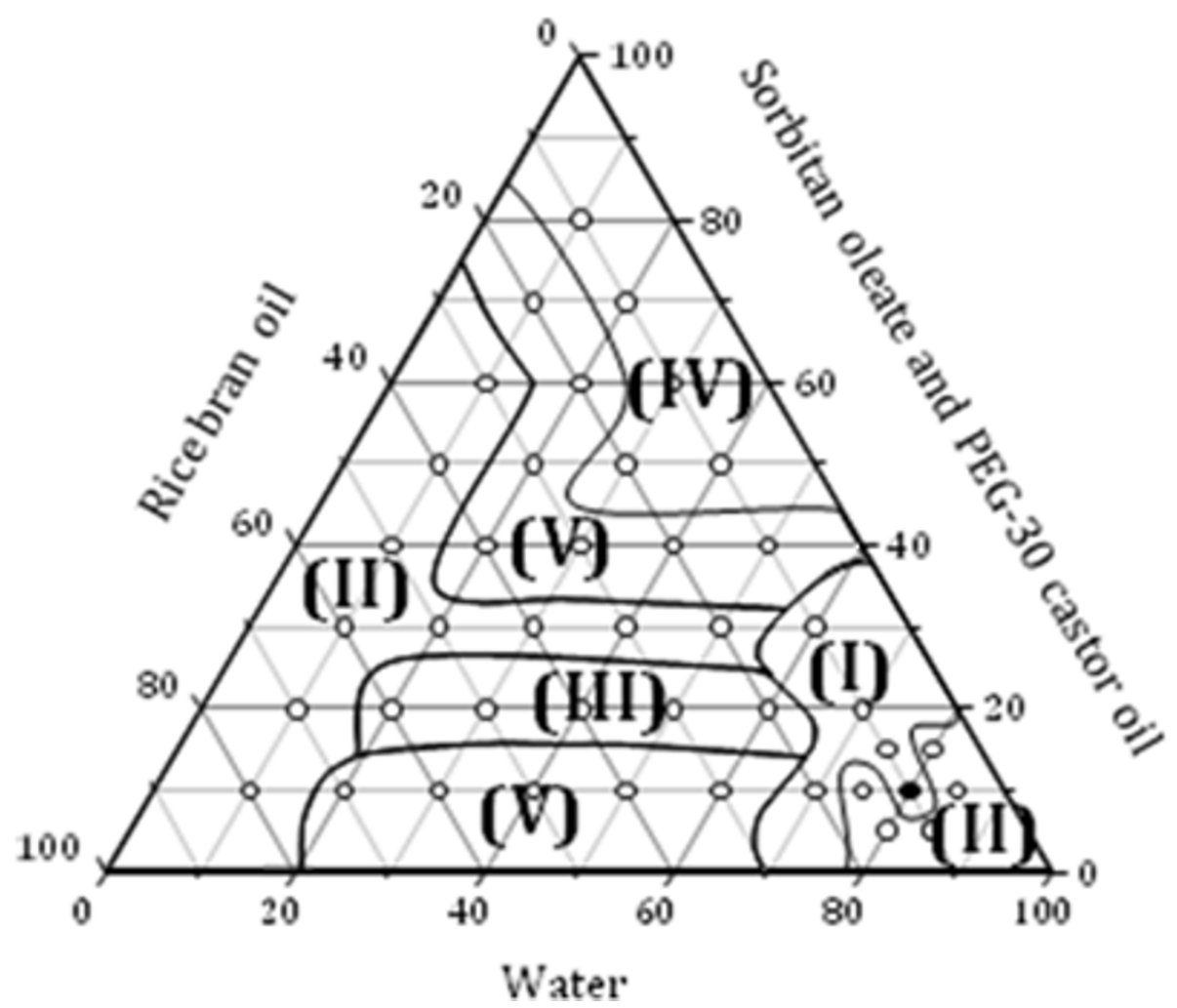

Figure 1 Phase diagram with rice bran oil, sorbitan oleate/PEG-30 castor oil and water. Region I: nanoemulsion, II: phase separation, III: gel phase, IV: W/O emulsions; V: O/W emulsions.

corroborating our results for temperatures of $5 \pm 2^{\circ} \mathrm{C}$ and $25 \pm 2^{\circ} \mathrm{C}$.

Low-energy emulsification is better at producing stable nanoemulsions than its higher energy counterpart. When nanoemulsions were prepared using a high pressure homogeniser, the droplet size was initially around $100 \mathrm{~nm}$; however, the particles increased in size after 30 days at either 25 or $4^{\circ} \mathrm{C}$. This phenomenon was attributed to the preparation method [28]. The low-energy emulsification method used in our study showed high stability with respect to the droplet size and polydispersity index.

\section{$\mathrm{pH}$ value determination}

Monitoring the $\mathrm{pH}$ value is important for determining the emulsions' stability because $\mathrm{pH}$ changes indicate the occurrence of chemical reactions that can compromise the quality of the final product. Emulsions produced with vegetable oils may experience a decrease in $\mathrm{pH}$ due to the hydrolysis of fatty acid esters into free fatty acid degradation products [29].

The nanoemulsions had stable $\mathrm{pH}$ values for almost all conditions tested (Figure 4). Only at a temperature of $40 \pm 2^{\circ} \mathrm{C}$ and 90 days of incubation was there a statistically significant decrease in the $\mathrm{pH}$ of the nanoemulsion. The high temperature might have destabilised the nanoemulsion by hydrolysis, but it did not affect the overall quality of the nanoemulsions because the $\mathrm{pH}$ values remained around $\mathrm{pH}$ 6.0, which is an acceptable, non-skin irritating $\mathrm{pH}$ value.

\section{Electrical conductivity}

The nanoemulsion showed changes in electrical conductivity at all storage conditions (Figure 5).

\section{Table 2 Composition of formulation characterised as nanoemulsions}

\begin{tabular}{cccc}
\hline Rice bran oil (\% w/w) & Sorbitan oleate/PEG-30 castor oil (\% w/w) & Purified water (\% w/w) & Droplet size (nm) \pm (Standard Deviation) \\
\hline 10.00 & 30.00 & 60.00 & $91 \pm 19$ \\
10.00 & 20.00 & 70.00 & $45 \pm 12$ \\
10.00 & 10.00 & 80.00 & $69 \pm 17$ \\
20.00 & 10.00 & 70.00 & $303 \pm 32$ \\
10.00 & 15.00 & 75.00 & $121 \pm 13$ \\
\hline
\end{tabular}




\section{Size Distribution by Intensity}

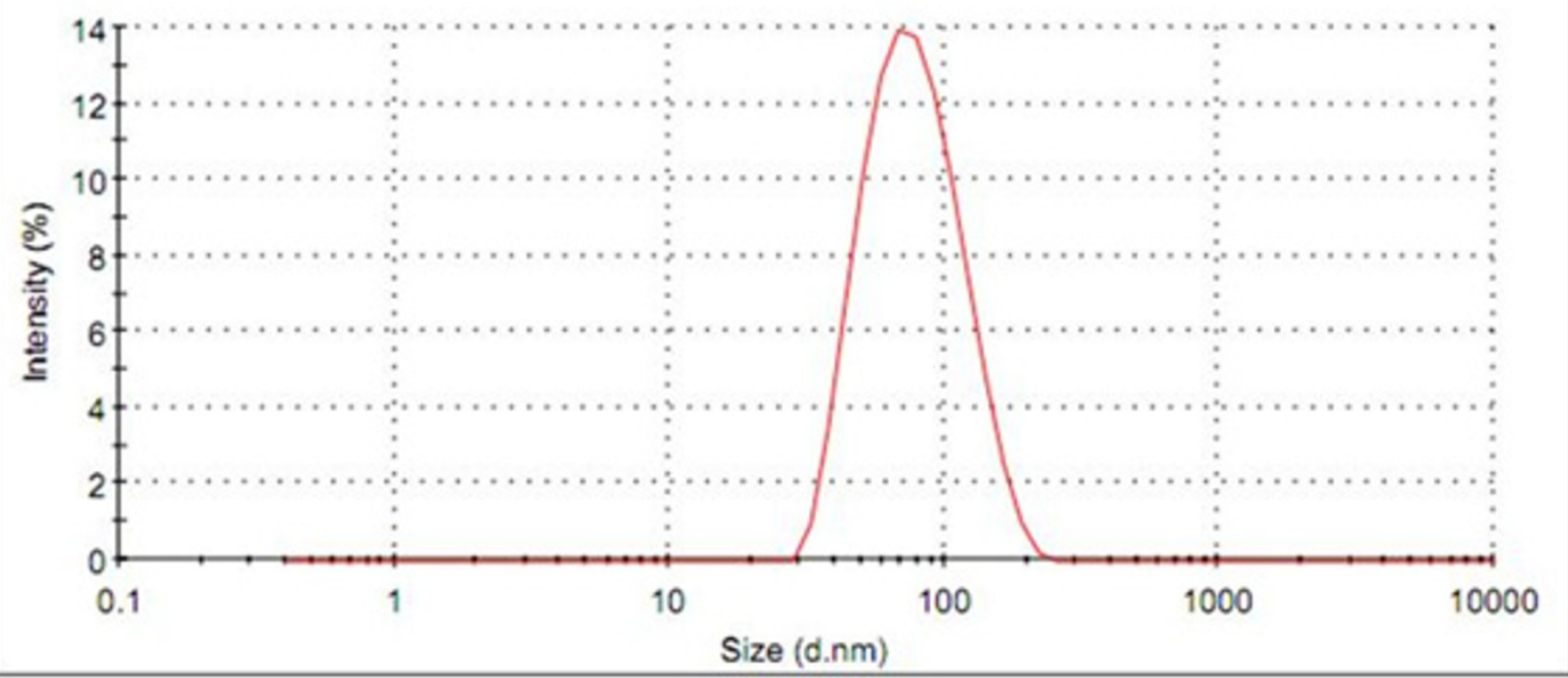

Figure 2 Droplet size distribution of nanoemulsions after 24 hours.

Changes in the electrical conductivity can indicate nanoemulsion instability and may influence the nanoemulsion droplet size [30]. In these studies, changes in electrical conductivity did not affect the nanoemulsion droplet size (Figure 3). It is difficult to assess the emulsion stability solely by electrical conductivity because the relationship between an increase in electrical conductivity and emulsion instability is not linear [31].

Thus, we could not conclusively determine the nanoemulsion's stability by this parameter. However, because the particle size and the $\mathrm{pH}$ value did not significantly change across different conditions, we considered our nanoemulsion to be stable. Nanoemulsion stability is a crucial parameter in determining the moisturising activity of the nanoemulsions in vivo.

\section{Irritant test in an organotypic model - HET-CAM (Hen's}

Egg Test on the Chorioallantoic Membrane)

Topical application products must have a low ocular/ mucous membrane and a low dermal irritation potential.

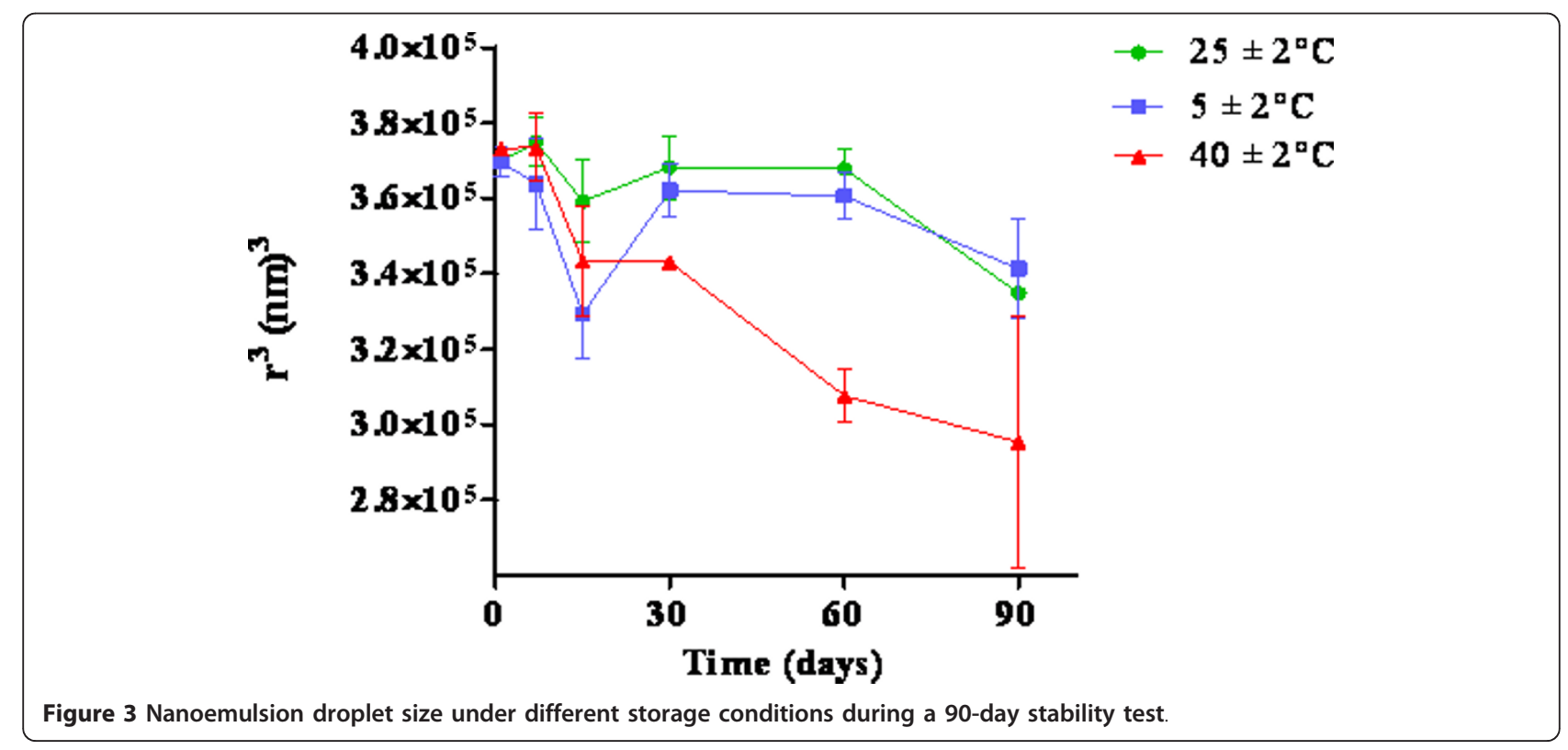




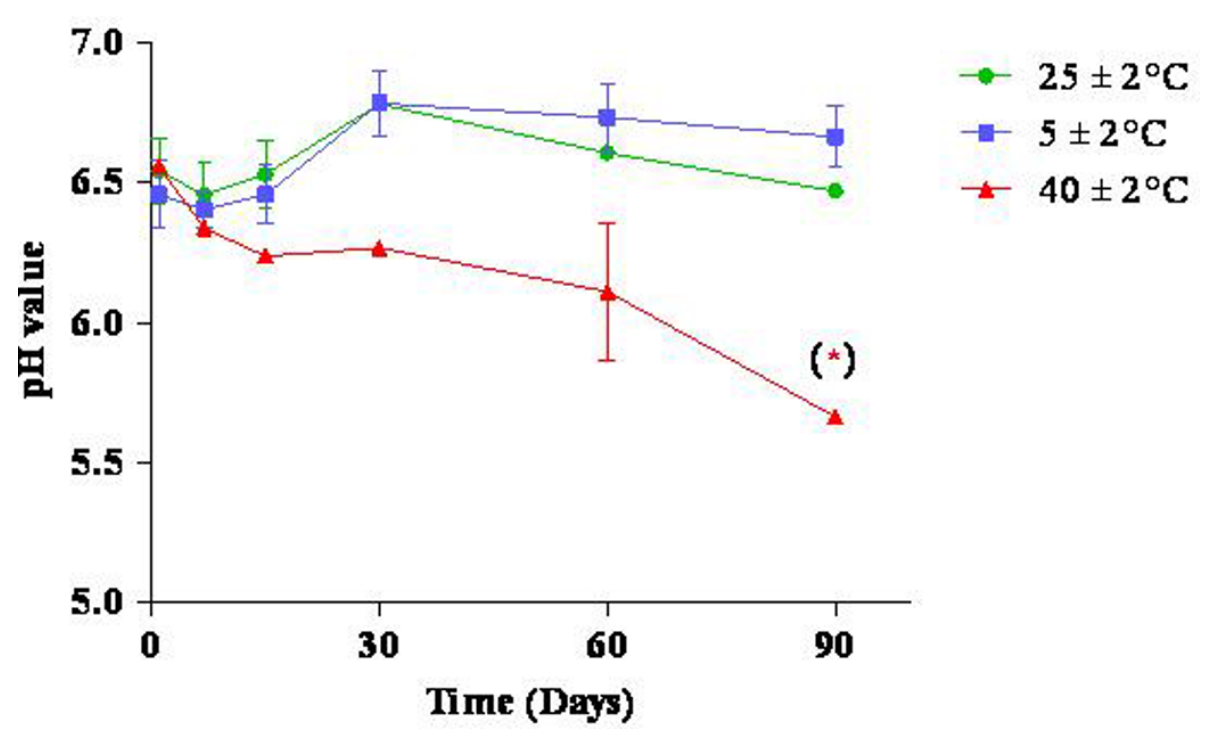

Figure $4 \mathrm{pH}$ values for nanoemulsions over time under different storage conditions.

The irritation potential depends on the concentration of the substance as well as the chemical composition and the $\mathrm{pH}$ of the formulation [25].

The HET-CAM test can help evaluate the irritation potential of substances in vitro and in vivo [32]. The CAM showed no signs of irritation after application of either the nanoemulsion or the negative control substance, so the nanoemulsion was therefore considered practically non-irritating. The surfactant solution by itself caused mild hyperaemia, which suggests that the presence of rice bran oil in the nanoemulsion may have protected the chorioallantoic membrane from the irritating effects of the surfactant solution (Table 3). The $\mathrm{pH}$ values were the same for all samples tested to eliminate $\mathrm{pH}$ as a variable in the HET-CAM results.

Previous studies showed that $\mathrm{O} / \mathrm{W}$ microemulsions containing linoleic acid were only barely irritating in the HET-CAM test, indicated by a slight discoloration of the chorioallantoic membrane [33]. The HET-CAM test showed that the nanoemulsion containing rice bran oil was essentially non-irritating.

\section{In vivo assessment}

The formulation composed of 10:10:80 (rice bran oil, surfactant blend and water) was chosen for the in vivo study due to its high stability and lack of irritation in

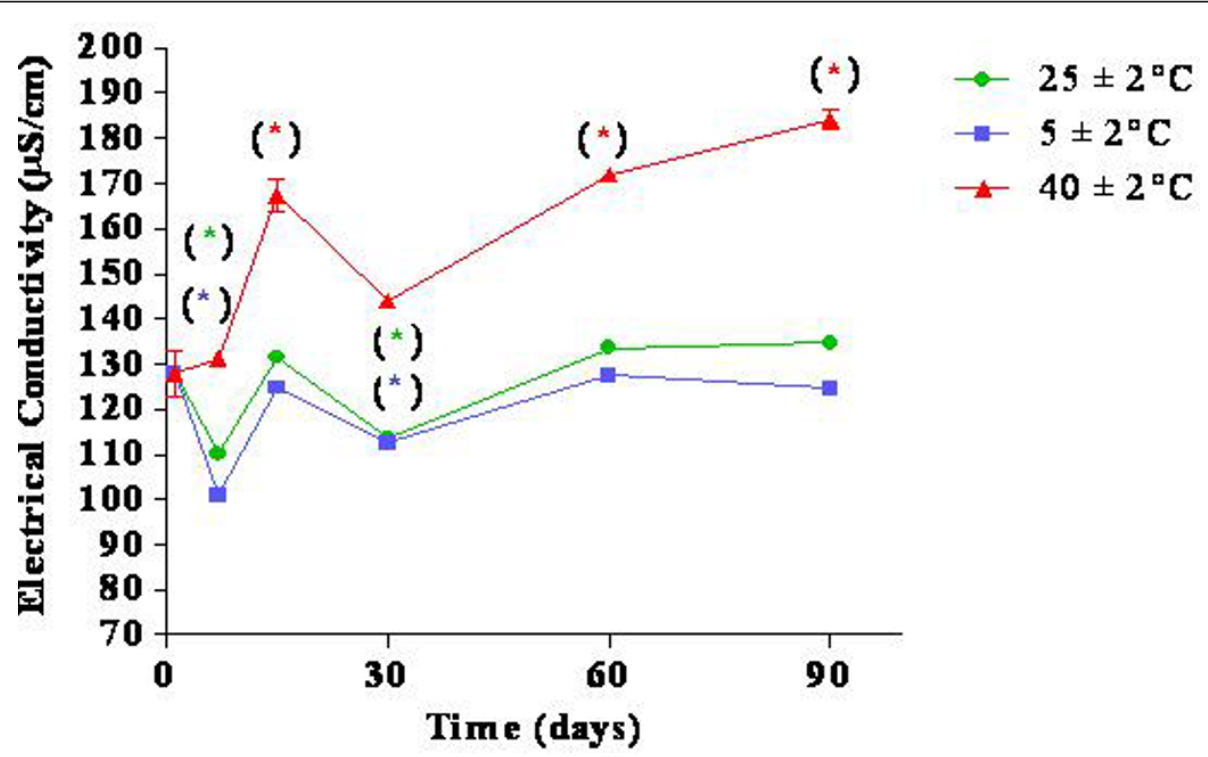

Figure 5 Electrical conductivity of nanoemulsions over time under different storage conditions. 
Table 3 Scores and assessments of irritation potential of nanoemulsions and surfactant solution tested in the chorioallantoic membrane test

\begin{tabular}{ccc}
\hline Formulation & Cumulative Score (average) & Assessment \\
\hline Nanoemulsion $^{1}$ & 0 & Practically none \\
Surfactant Solution $^{2}$ & 5 & Moderate \\
Positive control $^{3}$ & 15 & Strong \\
Negative control $^{4}$ & 0 & Practically none \\
\hline
\end{tabular}

${ }^{1} 10.00 \%$ of rice bran oil, $5.00 \%$ of sorbitan oleate, $5.00 \%$ of PEG-30 castor oil, $0.05 \%$ of BHT antioxidant, $0.50 \%$ of preservative and $79.45 \%$ of distilled water ${ }^{2} 5.00 \%$ of sorbitan oleate, $5.00 \%$ of PEG-30 castor oil and $90.00 \%$ of distilled water

${ }^{3} 10 \%$ of SLS and $90 \%$ of distilled water

${ }^{4}$ Saline solution

the HET-CAM test. This nanoemulsion formulation was applied to volunteers with either normal or affected skin (atopic dermatitis or psoriasis).

\section{Moisturising activity}

The moisturising activity of the stratum corneum is measured by skin capacitance. It is also an important tool in evaluating healthy and diseased skin such as patients with atopic dermatitis or psoriasis [34-36]. The moisturising variance in healthy volunteers increased both 30 and 60 minutes after nanoemulsion application and then decreased over the remainder of the study. Volunteers with atopic dermatitis or psoriasis showed increased moisturising variance in the first 30 minutes and maintained this increase up to 90 minutes after application. Then, the moisturising capacitance decreased after 90 minutes until the end of test (Figure 6). Skin affected by atopic dermatitis or psoriasis had a lower basal hydration value compared with healthy skin. People with these drying skin conditions have increased dryness in their skin outside of the regions with lesions [37-39]. The rice bran oil nanoemulsion increased the moisturising variance by about $38 \%$ in normal skin volunteers and by $30 \%$ in volunteers with atopic dermatitis or psoriasis, which is a satisfactory result because a high-quality commercial moisturiser only increased skin hydration by about $20 \%$ after 14 days of application [40]. These improved effects may be caused by the nanoemulsion droplets adhering to the skin and forming a dense film that inhibits water evaporation from the skin [41].

The rice bran oil nanoemulsion significantly increased the skin hydration in volunteers suffering from atopic dermatitis and psoriasis. Although the skin hydration measurements should be conducted over a longer period of time, 8 - 24 h, this study indicates that the nanoemulsion may provide long-term skin hydration.

\section{Oily Skin}

The oiliness values of nanoemulsion-treated skin increased considerably 30 minutes after treatment and then decreased in both the healthy and affected skin groups. The increase may be related to the amount of rice bran oil (10\%) in the formulation (Figure 7). Cosmetic emulsions form an oily layer on the skin that can protect the lipid barrier, which is desirable in dry skin conditions [42]. Therefore, the oiliness of the nanoemulsion may provide an alternative treatment for psoriasis.

\section{Skin $\mathrm{pH}$ determination}

Forearm skin testing is standard in most clinical studies of skin and has $\mathrm{pH}$ values in the range of 4.2 to 5.9 for both sexes [43]. The $\mathrm{pH}$ values of volunteers' skin tested during this study ranged from 4.9 to 5.2 after treatment for both groups. Thus, the $\mathrm{pH}$ changes due to the nanoemulsion

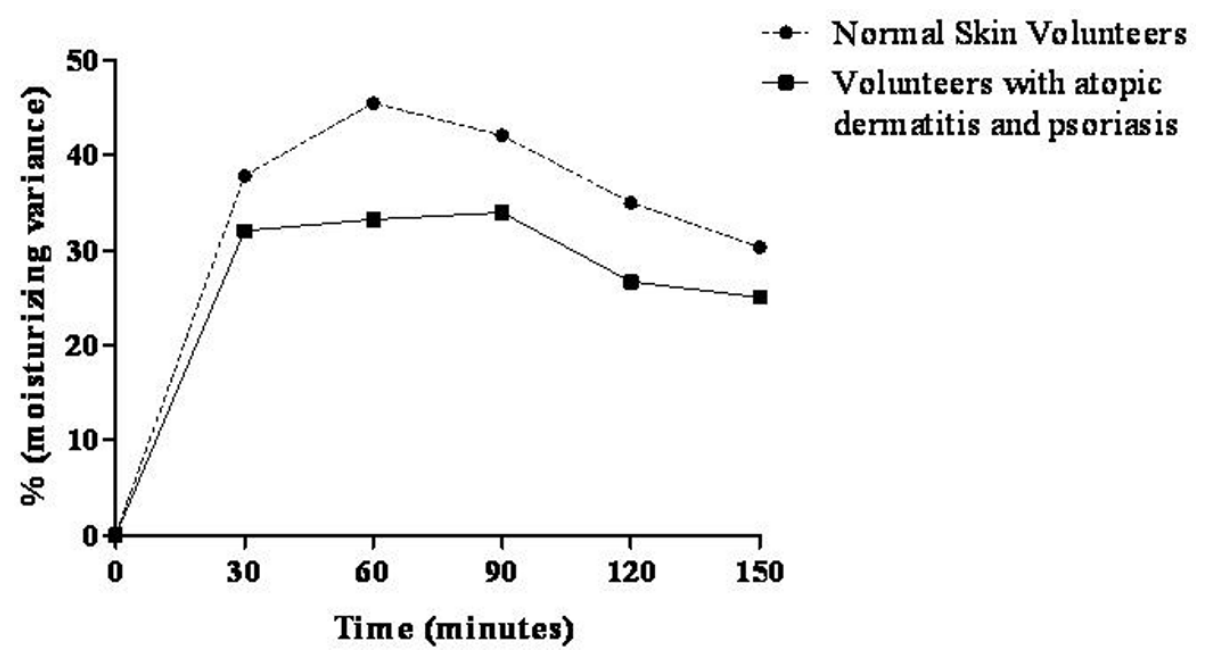

Figure 6 Moisturising variance after nanoemulsion treatment in volunteers with either normal skin or skin affected by atopic dermatitis or psoriasis. 


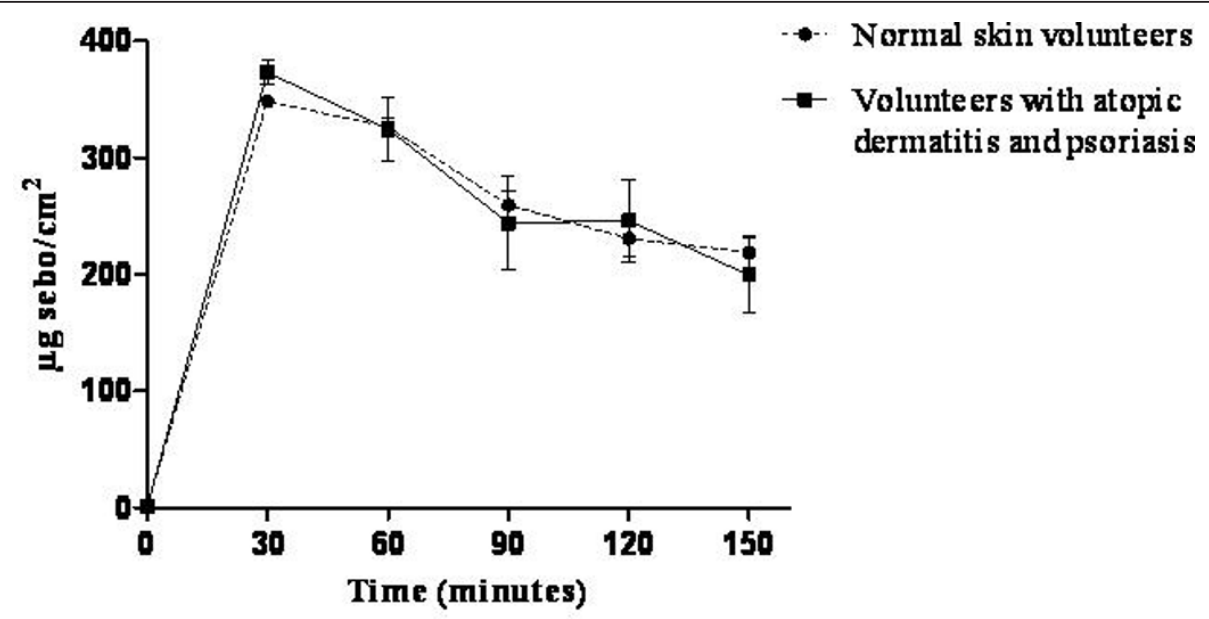

Figure 7 Oiliness of the skin after nanoemulsion treatment in volunteers with either normal skin or skin affected by atopic dermatitis or psoriasis.

were within the accepted $\mathrm{pH}$ range for forearm skin found in the literature, so this formulation does not significantly alter the skin $\mathrm{pH}$ (Figure 8).

\section{Conclusion}

The nanoemulsion developed in this study using the phase diagram method was composed of $10 \%$ rice bran oil, $10 \%$ surfactants sorbitan oleate/PEG-30 castor oil, $0.05 \%$ antioxidants and $0.50 \%$ preservatives formulated in distilled water. The nanoemulsion was stable during the period of study and was found to be practically non-irritating in the organotypic HET-CAM model. When applied to the skin of volunteers, the nanoemulsion increased the relative hydration of the skin, the skin oiliness and maintained normal skin $\mathrm{pH}$ values. This nanoemulsion could serve as an alternative treatment for skin diseases such as atopic dermatitis and psoriasis.

\section{Acknowledgements}

This work was supported by FAPESP (Fundação de Amparo à Pesquisa do Estado de São Paulo) protocol numbers: 2009/01922-3, 2008/10382-0, 2009/ 05774-9, 2009/07817-7 and 2010/09618-9, CNPq (Conselho Nacional de Desenvolvimento Científico e Tecnológico) and CAPES (Coordenação de Aperfeiçoamento de Pessoal de Nível Superior).

\section{Author details}

'Departamento de Ciências Farmacêuticas, Faculdade de Ciências

Farmacêuticas de Ribeirão Preto, Universidade de São Paulo, Ribeirão Preto,

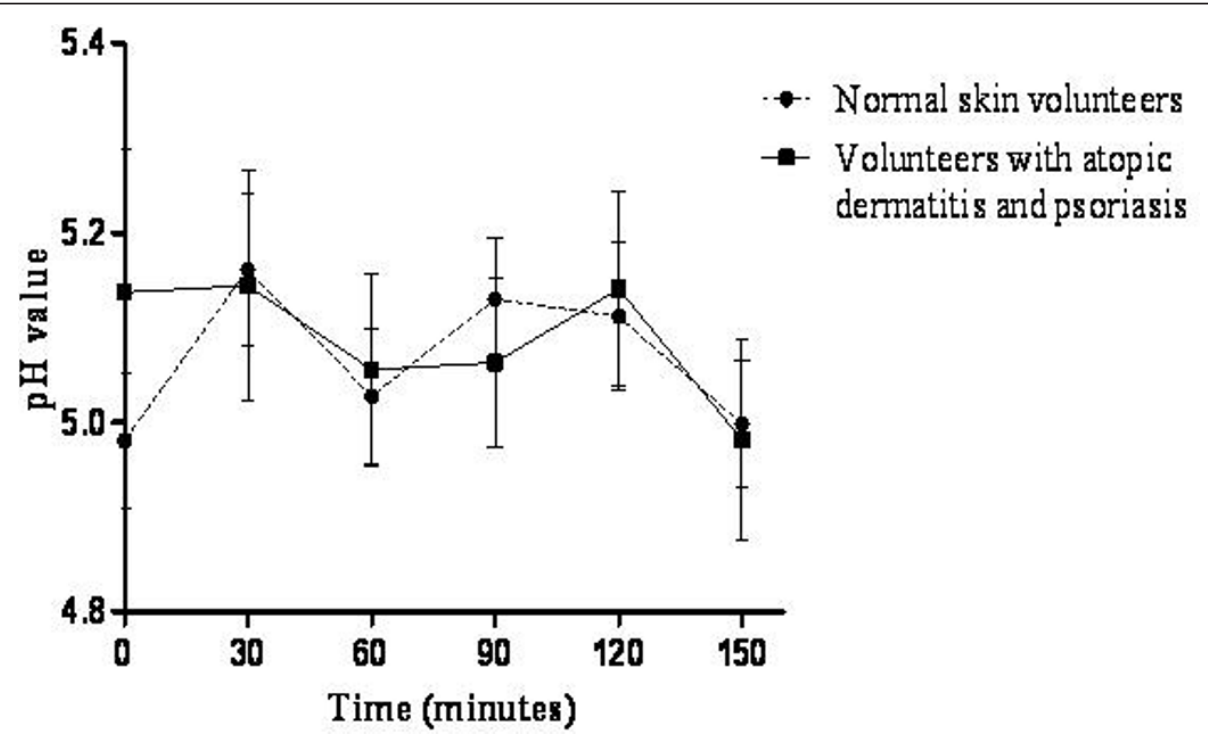

Figure 8 Skin pH value after nanoemulsion treatment in volunteers with normal skin or skin affected by atopic dermatitis or psoriasis. 
São Paulo, Brazil. 'Universidade de Ribeirão Preto, Ribeirão Preto, São Paulo, Brazil.

\section{Authors' contributions}

DSB selected the nanoemulsion composition through the phase diagram study. JB and DSB contributed to the stability study of formulation. NRM and DSB performed the irritant test in the organotypic model - HET-CAM. TAP and GSV performed the in vivo assessment. PARF and NRM guided the studies. The manuscript has been read and approved by all the authors.

\section{Competing interests}

The authors declare that they have no competing interests.

Received: 11 April 2011 Accepted: 28 September 2011 Published: 28 September 2011

\section{References}

1. Forgiarini A, Esquena J, González C, Solans C: Formation of nanoemulsions by low-energy emulsification methods at constant temperature. Langmuir 2001, 17(Suppl 7):2076-2083.

2. Thadros T, Izquierdo P, Esquena J, Solans C: Formation and stability of nanoemulsions. Advance in Colloid and Interface Science 2004, 108-109:303-318

3. Becher P: Emulsions: Theory and pratice Oxford: Oxford University Press; 2001.

4. Solans C, Izquierdo P, Nolla J, Azemar N, Garcia-Celma MJ: Nano-emulsions. Current Opinion in Colloid and Interface Science 2005, 10(Suppl 3-4):102-110.

5. Taylor P: Ostwald ripening in emulsions. Colloids and Surfaces A Physicochemical and Engineering Aspects 1995, 99(Suppl 2-3):175-185.

6. Lifshitz IM, Slesov $\mathrm{W}$ : The kinetics of precipitation from supersaturated solid solutions. Journal of Physics and Chemistry of Solids 1961, 19(Suppl 1/ 2):35-50.

7. Wagner C: Theory of precipitate change by redissolution. Zeitschrift Fur Elektrochemie 1961, 65:581-591.

8. Abdulrazik M, Tamilvanan S, Khoury K, Benita S: Ocular delivery of ciclosporin A. Il. Effect of submicron emulsion's surface charge on ocular distribution of topical cyclosporin. STP Pharma Science 2001, 11:427-432.

9. Gershanik T, Benita S: Positively charged self-emulsifying oil formulation for improving oral biovailability of progesterone. Pharmaceutical Development and Technology 1996, 1:147-157.

10. Klang S, Benita S: Design and evaluation of submicron emulsion as colloidal drug carriers for intravenous administration. In Submicron emulsions in drug targeting and delivery Edited by: Benita S 1998, 119-152.

11. Solans C, Esquena J, Forgiarini A, Izquierdo P, Morales D, Uson N, Azemar N, Garcia-Celma MJ: Surfactants in solution: Fundamentals and applications. In Surfactant Science Series. New York Edited by: Mittal KL, Shah D, Dekker M 2002.

12. Lilitchan S, Tangprawat C, Aryusuk K, Krisnangkura S, Chokmoh S, Krisnangkura K: Partial extraction method for the rapid analysis of total lipids and gamma-oryzanol contents in rice bran. Food Chemistry 2008, 106:752-759.

13. Coppini D, Paganizzi $P$, Santi $P$, Ghirardini A: Capacitá protettiva Nei confronti delle radiazioni Solari di derivati di origine vegetable. Cosmetic News 2001, 136:15-20.

14. Patel M, Naik SN: Gamma-oryzanol from rice bran oil: a review. Journal of Scientific \& industrial Research 2004, 63:569-578.

15. Lerma-García MJ, Herrero-Martínez JM, Simó-Alfonso EF, Mendonça CRB, Ramis-Ramos G: Composition industrial processing and applications of rice bran gamma-oryzanol. Food Chemistry 2009, 115:389-404.

16. Leung DYM: Atopic dermatitis: new insights and opportunities for therapeutic intervention. Journal of Allergy and Clinical Immunology 2000, 105(Suppl 5):860-876.

17. Leung DYM, Boguniewicz M, Howell MD, Nomura I, Hamid QA: New insights into atopic dermatitis. Journal of Clinical Investigation 2004, 113(Suppl 5):651-657

18. Lodén M: The skin barrier and use of moisturizers in atopic dermatitis. Clinics in Dermatology 2003, 21(Suppl 2):145-157.

19. Mchenry PM, Williams HC, Bingham EA: Management of atopic eczema: Joint Workshop of the British Association of Dermatologists and the Research Unit of the Royal College of Physicians of London. British Medical Journal 1995, 310:843-847.

20. Rudolph R, Kownatzi E: Corneometric, sebumetric and TEWL, measurements following the cleaning of atopic skin with a urea emulsion versus a detergent cleanser. Contact Dermatitis 2004, 50:354-358.
21. Alanen E, Nuutinen J, Nicklén K: Measurement of hydration in the stratum corneum with the MoistureMeter and comparison with the Corneometer. Skin Research and Technology 2004, 10(Suppl 1):32-7.

22. Steiling $W$, Bracher M, Courtellemont P, De Silva O: The HET-CAM, a useful in vitro assay for assessing the eye irritation properties of cosmetic formulations and ingredients. Toxicology In vitro 1999, 12:375-384.

23. Izquierdo P, Feng J, Esquena J, Tadros TF, Dederen JC, Garcia MJ, Azemar N, Solans $C$ : The influence of surfactant mixing ratio on nano-emulsion formation by the pit method. Journal of Colloid and Interface Science 2005, 285:388-394.

24. Luepke NP: Hen's egg chorioallantoic membrane test for irritation potential. Food and Chemical Toxicology 1985, 23:287-291.

25. Mehling A, Kleber M, Hensen H: Comparative studies on the ocular and dermal irritationpotential of surfactants. Food and Chemical Toxicology 2007, 45:747-758.

26. Jafari SM, Assadpoor E, He Y, Bhandari B: Re-coalescence of emulsion droplets during high-energy emulsification. Food Hydrocolloids 2008, 22:1191-1202.

27. Shakeel F, Baboota S, Ahuja A, Ali J, Faisal MS, Shafiq S: Stability evaluation of celecoxib nanoemulsion containing Tween 80 . Thai Journal of Pharmaceutical Science 2008, 32:4-9.

28. Yuan Y, Gao Y, Zhao J, Mao L: Characterization and stability evaluation of b-carotene nanoemulsions prepared by high pressure homogenization under various emulsifying conditions. FoodResearchlnternational 2008, 41:61-68.

29. Martini E: Nanoemulsões catiônicas como sistemas de liberação de oligonucleotídeos: formulação e caracterização físico-química. Dissertação (mestrado). Universidade do Rio Grande do Sul, Porto Alegre; 2005.

30. ANVISA (Agência Nacional de Vigilância Sanitária): Guia de Estabilidade de Produtos Cosméticos. Brasília 2004.

31. Masmoud $H$, Dréau YL, Piccerelle P, Kister J: The evaluation of cosmetic and pharmaceutical emulsions aging process using classical techniques and a new method: FTIR. International Journal of Pharmaceutics 2005, 289:117-131

32. Wilson TD, Steck WF: A modified HET-CAM assay approach to the assessment of anti-irritant properties of plant extracts. Food and ChemicalToxicology 2000, 38::867-872.

33. Goebel ASB, Knie U, Abels C, Wohlrab J, Neubert RHH: Dermal targeting using colloidal carrier systems with linoleic acid. European Journal of Pharmaceutics and Biopharmaceutics 2010, 75(Suppl2):162-172.

34. Agache $P$, Mary $S$, Muret $P$, Matta AM, Humbert P: Assessment of the water content of the stratum corneum using a sorption-desorption test. Dermatology 2001, 202:308-313.

35. Crowther JM, Sieg A, Blenkiron P, Marcott C, Matts PJ, Kaczvinskyà JR, Rawlings AV: Measuring the effects of topical moisturizers on changes in stratum corneum thickness, water gradients and hydration in vivo. British Journal of Dermatology 2008, 159:567-577.

36. Rim JH, Jo SJ, Park JY, Park BD, Youn Jl: Electrical measurement of moisturizing effect on skin hydration and barrier function in psoriasis patients. Clinical and Experimental Dermatology 2005, 30:409-413.

37. Wissing SA, Muller RH: Cosmetic applications for solid lipid nanoparticles (SLN). International Journal of Pharmaceutics 2003, 254(Suppl 1):65-68.

38. Zhou H, Yue Y, Liu G, Li Y, Zhang J, Gong Q, Van Z, Duan M: Preparation and characterization of a lecithin nanoemulsion as a topical delivery system. Nanoscale Research Letters 2010, 5:224-230

39. Bowcock AM, Cookson WOCM: The genetics of psoriasis, psoriatic arthritis and atopic dermatitis. Journal Human Molecular Genetics 2004, 13(Suppl 1): R43-R55

40. Fluhr JW, Cavallotti C, Berardesca E: Emollients, moisturizers, and keratolytic agents in psoriasis. Clinics in Dermatology 2008, 26:380-386.

41. Proksch E: The role of emollients in the management of diseases with chronic dry skin. Skin Pharmacology and Physiology 2008, 21:75-80.

42. Machado M, Bronze MR, Ribeiro H: New cosmetic emulsions for dry skin. Journal of Cosmetic Dermatology 2007, 6:239-242.

43. Ehlers C, Ivens UI, Moller ML, Senderovitz T, Serup J: Females have lower skin surface $\mathrm{pH}$ than men. A study on the influence of gender, forearm site variation, right/left difference and time of the day on the skin surface pH. Skin Research and Technology 2001, 7:90-94.

doi:10.1186/1477-3155-9-44

Cite this article as: Bernardi et al.: Formation and stability of oil-in-water nanoemulsions containing rice bran oil: in vitro and in vivo assessments. Journal of Nanobiotechnology 2011 9:44. 